\title{
Management of Blunt Splenic Injuries in the Setting of Low Trauma Volume: Challenges and Perspectives
}

El-Rifai AY ${ }^{1}$, Abi-Saad $\mathbf{G}^{1}$, Qaraqe $\mathbf{T}^{1}$, Farhat $\mathbf{H}^{1}$, El-Hechi $\mathbf{M}^{1}$, Hallal $\mathbf{A}^{1^{*}}$, Jaafar $\mathbf{R}^{1}$, El-Merhi $\mathbf{F}^{2}$, Saade $\mathbf{C}^{2}$, Haydar $\mathbf{A}^{2}$ and Nicholas Batley ${ }^{3}$

${ }^{1}$ Department of Surgery, Division of General Surgery, American University of Beirut Medical Center, Beirut, Lebanon

${ }^{2}$ Department of Diagnostic Radiology, American University of Beirut Medical Center, Beirut, Lebanon

${ }^{3}$ Department of Emergency Medicine, American University of Beirut Medical Center, Beirut, Lebanon

*Corresponding author: Ali Hallal, Assistant Professor of Clinical Surgery, Department of Surgery, Division of General Surgery, American University of Beirut Medical Centre. Beirut, P.O. Box-110236, 11072020, Lebanon, Tel: 009611 350000; E-mail: ah05@aub.edu.lb

Rec Date: June 29, 2018; Acc Date: August 22, 2018; Pub Date: August 24, 2018

Copyright: $\odot 2018$ El-Rifai AY, et al. This is an open-access article distributed under the terms of the Creative Commons Attribution License, which permits unrestricted use, distribution, and reproduction in any medium, provided the original author and source are credited.

\begin{abstract}
The management of Blunt Splenic Injury (BSI) has changed overtime and Non Operative Management (NOM) is the standard of care. This study reports the experience of the American University of Beirut Medical Center (AUBMC) in the management of (BSI) since the establishment of a trauma team in 2010.
\end{abstract}

Keywords: Blunt splenic injury; Splenic angiography; Non-operative management; Embolization, Contrast blush

\section{Introduction}

The management of splenic injury has changed significantly over the last decades. Non-operative management (NOM) of Blunt Splenic Injuries (BSI) has been gaining popularity and had become the standard of care in hemodynamically stable patients [1-3]. NOM comprises observation and monitoring as well as angiography and embolization with the objective to preserve the spleen because of its recognition as a vital organ in immunity [4]. However, some patients with splenic injury will fail NOM. The failure rate reported in some studies range from $10 \%$ to $38 \%$ [5-7]. The last decade has shown a steady improvement in the non-operative failure rate down to a rate of $3 \%$ with the addition of protocols clarifying the indications for splenic artery angiography and embolization [8-10]. While embolization of AAST grade IV-V BSI or any injury with evidence of contrast extravasation is recommended in most case [9-13], the management of grade III splenic injury is still controversial with both observation and angiography considered as acceptable options $[10,11,14]$. The purpose of this study is to describe the cases of BSI admitted to American University of Beirut Medical Center, a major tertiary care center in Lebanon, and to highlight the management strategy patients with BSI since the establishment of the trauma team in 2010. Furthermore, patient outcomes will be compared to regional [15-17]and international benchmarks.

\section{Material and Methods}

The study is a retrospective observational study of patients admitted to the AUBMC with BSI over a six-year period from Jan 2010 at the time of the inception of the trauma team till August 2016. Access to the charts was obtained after attaining approval from the Institutional Review Board (IRB) at the AUBMC. The study included all patients older than 17 years with blunt trauma to the abdomen with documented injury to the spleen.
Patients with a discharge diagnosis of splenic injury or splenic trauma were identified and cross-referenced with the radiology database to ensure completeness of the data. Data collected and analyzed included demographics, mechanism of injury, Injury Severity Score (ISS), physiologic parameters on arrival, Glasgow Coma Scale (GCS), transfusions requirement on day two and day seven, all hospital complications, Intensive Care Unit (ICU) length of stay, hospital length of stay and mortality. Splenic injury was graded as per the American Association for the Surgery of Trauma (AAST) Organ Injury Scale [18].

Patients were divided based on the management strategy into three groups. The first group is the Non-Operative Management (NOM) group defined as patients who were admitted to ICU for initial observation and who might have had angiography for contrast blush on CT scan but did not proceed to embolization, the second group includes patients with initial trans-arterial embolization (TAE) of the splenic artery and the third group is the surgery group defined as Immediate Operative Management (IOM) patients who underwent immediate splenectomy or splenorraphy without a trial of observation.

Failure of non-operative management (f-NOM) is defined as any patient who was in the ICU or on the ward with planned NOM who needed either TAE or surgery at any point in time during their hospital stay. The study analyzes the frequency and indication for splenectomy, the utilization of splenic embolization and the risk factors that are associated with f-NOM. The study also analyzed the morbidity and mortality of each management strategy (Figure 1).

\section{Statistical Analysis}

Statistical analysis was performed with SPSS Version 24.0. Data were expressed as means \pm standard deviation (SD), or the mode and range. Comparisons between groups were performed using one-way ANOVA and non-parametric tests. A value of $p<0.05$ was considered statistically significant. 


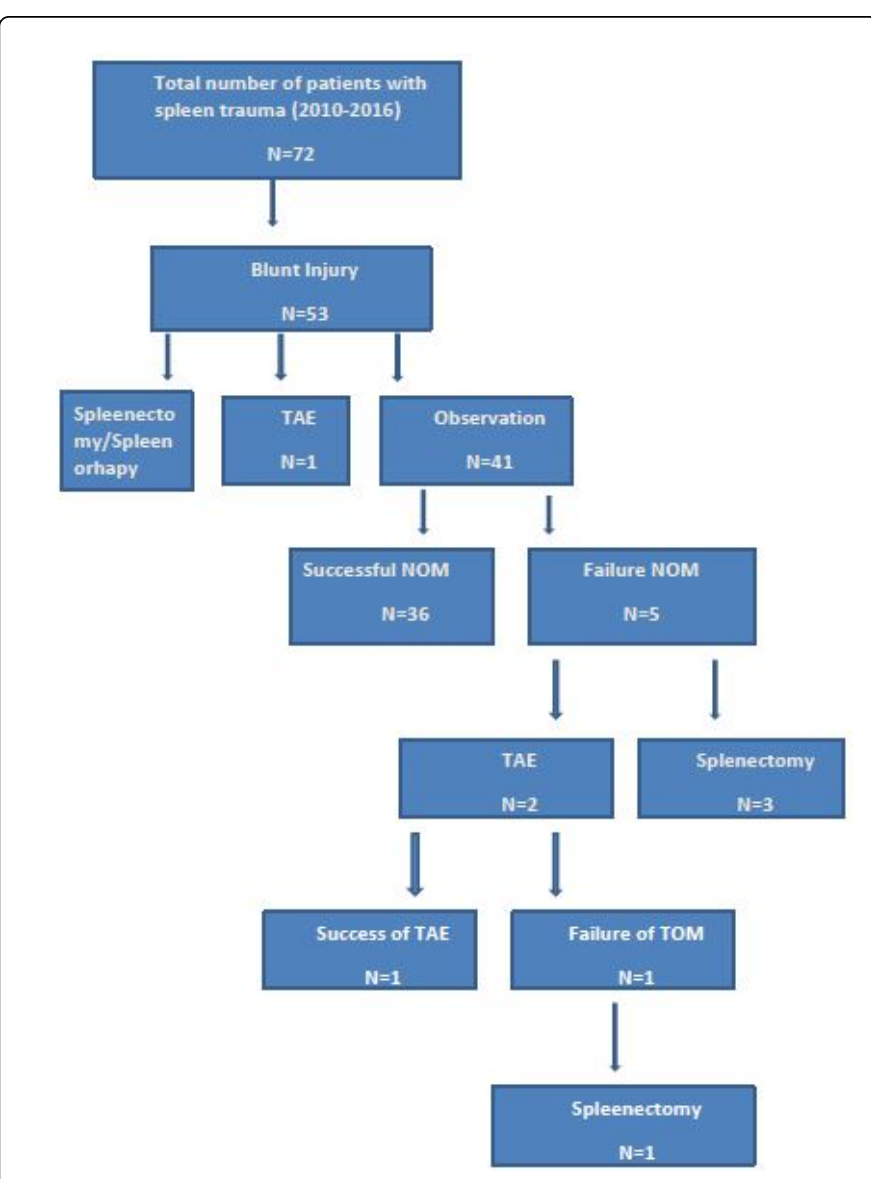

Figure 1: Patients admitted with blunt splenic injury to the AUBMC between 2010 and 2016.

\section{Results and Discussion}

The male predominance of our trauma patients, the pattern of mechanism of injuries and their prevalence as well as the distribution of injury AAST grades were similar to what is reported in the literature $[2,5,13]$. The immediate splenectomy rate in our study was $20 \%$ and the total splenectomy rate was $26 \%$. The rate of immediate splenectomy in various institutional and multi-institutional studies varies between $10 \%$ to $30 \%$ of BSI $[2,5,13,19,20]$. Patients who had IOM were older had a higher ISS, a higher percentage of high grade (IV \& V) splenic injuries, lower GCS, lower admission Systolic Blood Pressure. As expected the IOM group received more PRBC transfusions at two days and seven days than the NOM group $(\mathrm{p}<0.001)$, and this is attributed to their corresponding higher severity of injury. The EAST multi-institutional spleen study [5] reported an average of 8.4 units of PRBC transfused within 24 hours for the immediate splenectomy as opposed to 1.2 units in the successfully observed group.

We identified eight cases with contrast blush on initial CT. Five patients had immediate splenectomy and only one patient with grade III and two patients with grade IV injuries proceeded to angiography. Only one patient with grade IV injury showed active extravasation on angiography and proceeded to embolization. The other two patients did not have embolization and had a successful outcome. There is considerable variation in the management of BSI particularly in high grade injury [21]. Miller et al. [9] used a protocol requiring angiography and embolization for all high grade injuries without an indication for immediate operation or angiography and showed a a statistically significant decrease in failure rate down to $5 \%$ compared to a failure rate of $15 \%$ when compared to a historical control from the same institution.

Controversy still persist regarding the benefit of angioembolization of grade III and IV injuries. In their retrospective study Bhullar et al. [11] analyzed two groups of patients $(n=539)$ with BSI who were managed with either observation or angioembolization. Their results showed that there was no difference in the failure rate for patients with grade I to III splenic injuries but there was clearly a significant decrease in the failure of non operative treatment with the addition of angioembolization for grade IV and V injuries. Harbrecht et al. [22,23] reported in their retrospective studies that there is an increase in the detection of BSI over time due to technological advancement and as a consequence the success attributed to angioembolization in studies using historical control may be the result of a time-dependent increase in the detection of a BSI which most likely would not have bled as opposed to the effect of angioembolization itself.

The recent splenic injury outcomes multi-institutional trial [24] also showed no statistical difference in the splenic salvage rate between patients admitted with BSI grade II to IV who had angioembolization when compared to those who did not. The 2016 updates of The Western Trauma Association [1] consider angiography in patients with grade III injury to be still controversial but recommends performing it in patients with grade III injury and a contrast blush detected on CT. On the contrary, splenic artery angioembolization is strongly recommended in patients with grade IV and V injuries with or without blush based on emergent recent evidence that showed that patients with high grade splenic injuries who undergo embolization had a high success rate $[9,10,12]$ while those who did not have embolization had a higher rate of failure rate reaching $26 \%$ [11] and ultimately needed another intervention which is usually a splenectomy [12].

As our data shows, two patients who had evidence of pseudoaneurysm on CT had angiography but did not proceed to embolization as their angiography was normal. Without a clear institutional protocol we faced a challenging task in proceeding with embolization of Grade III or IV splenic injury with a contrast blush in the setting of a normal angiography and stable patient especially when complications of splenic embolization were taken into consideration [25]. Furthermore some studies have shown that contrast blushes and pseudoaneurysms can be followed safely with a high rate of spontaneous resolution without embolization.

The failure for the different AAST grade in our study was $0 \%$ for grade I, $9 \%$ for grade II, $15.4 \%$ for grade III and $22 \%$ for grade IV. In one of the largest multicenter studies, the the failure rate of grade III to V BSI were $19.6 \%, 33 \%$, and $75 \%$ respectively [5]. The NOM failure rate for high grade IV-V injuries reported in clinical series with more than 100 patients ranges from $3 \%$ till $38 \%[6,9-11]$. The failure rate across all grades in our study was $12.1 \%$ which is comparable to what has been reported by large multi-institutional studies [26-29]. Our analysis has shown that only age and the volume of blood transfusion after day seven were found to differ statistically between the s-NOM and f-NOM while ISS, GCS and comorbidities were not associated with f-NOM. Furthermore there was no difference in the length of ICU and hospital stay between the two groups. Several reports have investigated prognostic variables for failure of NOM with varying 
Page 3 of 7

results [30,31]; the variability in the results is attributed to the heteregoeneity of the studies.

Of the five cases that failed NOM in this study none failed in the first 24 hours. Failure within the first 24 hours is attributed to a an unrecognized need for laparotomy and inappropriate selection for NOM [32,33]. Peitzman et al. [34] reported that $61 \%$ of patient failed with the first 24 hours with the majority of these patients having a clear indication for IOM rather than NOM. The mean time to failure in our study was 6 days. Four of the five patients that failed bled; two of the 4 patients had hemodynamic compromise and all four needed splenectomy. The fifth patient had evidence of progression in his splenic injury and was successfully salvaged with arterial embolization. Only one patient had hemodynamic instability on admission but responded quickly to resuscitation but then bled 4 days later. Three out of the remaining hemodynamically stable patients on arrival had some findings that should have alerted us to the high likelihood of failure of NOM. One patient had a large hemoperitoneum and a Grade III splenic injury, one patient had a missed contrast extravasation from a Grade III splenic laceration and one patient had a small pseudoaneurysm detected on CT in a grade IV splenic laceration. One patient who failed NOM died. This patient had an ISS of 41 and developed refractory hypoxemia secondary to severe ARDS. with a mortality rate in that group reaching $20 \%$.

There are several limitations in our study. As a retrospective study abstracting data from chart reviews introduce a systemic error in the accuracy of the data utilized. Without having an institutional protocol for splenic injuries the management decisions and consequently the corresponding outcomes were based on clinical judgment which raises the concern for a selection bias.

A total of 72 patients with splenic injury were admitted to the AUBMC between 2010-2016. Nineteen patients were excluded from the study because they had penetrating injuries leaving 53 patients in the study group. The mean age of the patients was 34 years (18-85). Only $13 \%$ of the patients were older than 55 years. Males constituted $81 \%$ and a quarter of the patients had one or more comorbidity. The age and gender was not different among the different AAST groups. The most common mechanism of injury was related to motor vehicle collisions (62\%), followed by falls (26\%), while blasts accounted for $6 \%$ and other mechanisms accounted for the remaining $6 \%$ (Table 1 ).

\begin{tabular}{|c|c|c|c|c|c|c|}
\hline \multirow{2}{*}{ Variables } & \multicolumn{6}{|c|}{ AAST Grade } \\
\hline & $I(n=8)$ & II (n=13) & III (n=16) & IV (n=12) & $V(n=4)$ & P-value \\
\hline Age Mean (S.D) & $30.1(15.2)$ & $31.9(15.7)$ & $34.8(20)$ & $25.8(18)$ & 39 (28.9) & 0.92 \\
\hline Age>55 (\%) & $0(0)$ & $2(15.4)$ & $3(18.8)$ & $1(8.3)$ & $1(25)$ & 0.665 \\
\hline Male (\%) & $7(87.5)$ & $11(84.6)$ & $12(75)$ & $11(91.7)$ & $2(50)$ & 0.402 \\
\hline Female (\%) & $1(12.5)$ & $2(15.4)$ & $4(25)$ & $1(8.3)$ & $2(50)$ & 0.402 \\
\hline Comorbidities (\%) & $1(12.5)$ & $2(15.4)$ & $6(37.5)$ & $3(25)$ & $1(25)$ & 0.624 \\
\hline \multicolumn{7}{|c|}{ Mechanism of injury } \\
\hline Motor Vehicle Collision & $6(75)$ & $10(76.9)$ & $7(43.8)$ & $9(75.0)$ & $1(25)$ & \\
\hline Fall & $1(12.5)$ & $3(23.1)$ & $5(31.3)$ & $2(16.7)$ & $3(75)$ & \\
\hline Blast & $1(12.5)$ & $0(0)$ & $2(12.5)$ & $0(0)$ & $0(0)$ & \\
\hline Other & $0(0)$ & $0(0)$ & $2(12.5)$ & $1(8.3)$ & $0(0)$ & \\
\hline
\end{tabular}

Table 1: Baseline characteristics per AAST grade.

The AAST grade of injuries was distributed as follows: $8(15 \%)$ patients had Grade I injury, 13 (24\%) patients had Grade II, 16 (30\%) patients had Grade III, 12 (23\%) patients had Grade IV, and only 4 (8\%) patients had Grade V. The mean ISS increased with severity of splenic injury grade reaching statistical significance (p-value 0.04 ) with a mean ISS of 18, range (4-41). (Table 2). Splenectomy on initial admission to the hospital was performed in 10 patients out of the 53 (19\%) while one (1.8\%) patient had splenorraphy. The rate of immediate operative interventions was $21 \%$. Non Operative Management (NOM) was attempted in 41 (77\%) patients, one patient had TAE from the onset. The management plan varied across AAST injury grade as described in (Table 2).

All Grade I injuries (8 cases, 100\%) had no direct interventions and were managed with frequent monitoring and observation, $11(85 \%)$ out 13 Grade II injuries had NOM while 2 cases had splenectomy due to evidence of mesenteric bleeding in one case and hemodynamic instability in the other one. Thirteen (81\%) out of 16 patients with Grade III injuries were observed while 3 (19\%) patients had an operative intervention.

Two patients had splenectomy, one for concomitant diaphragmatic rupture and one for splenic laceration in the setting of suspected splenic lymphoma. One case underwent laparotomy for hemodynamic instability but a splenorraphy was deemed safe. Nine (75\%) out of 12 patients with Grade IV injuries were observed while 2 cases underwent splenectomy due to hemodynamic instability and one case underwent TAE. Finally, all four patients with Grade V injuries had immediate splenectomy (Table 2). 
Citation: El-Rifai AY, Abi-Saad G, Qaraqe T, Farhat H, El-Hechi M, et al. (2018) Management of Blunt Splenic Injuries in the Setting of Low Trauma Volume: Challenges and Perspectives. J Trauma Treat 7: 430. doi:10.4172/2167-1222.1000430

Page 4 of 7

\begin{tabular}{|l|l|l|l|l|l|}
\hline Injury Grade & I (n=8) & II (n=13) & III (n=16) & IV (n=12) & V (n=4) \\
\hline Percentage of population (\%) & 15 & 24 & 30 & 23 & 8 \\
\hline ISS mean & $13.5(10.1)$ & $18.5(10)$ & $17.7(9.1)$ & $20.3(8.6)$ & $28.3(7.5)$ \\
\hline (Standard Deviation) & $13.5(10.1)$ & $18.5(10)$ & $17.7(9.1)$ & $20.3(8.6)$ & $28.3(7.5)$ \\
\hline Management plan & \multicolumn{5}{|l}{} \\
\hline Observation N (\%) & $8(100)$ & $11(84.6)$ & $13(81.3)$ & $9(75)$ & - \\
\hline Splenectomy N (\%) & - & $2(15.4)$ & $2(12.5)$ & $2(16.7)$ & $4(100)$ \\
\hline Contrast Blush N (\%) & - & - & $1(6)$ & $4(33)$ & $3(75)$ \\
\hline TAE N (\%) & - & - & - & $1(8.3)$ & - \\
\hline Splenorrhaphy N (\%) & - & - & - & - \\
\hline
\end{tabular}

Table 2: Initial management plan per AAST injury grade.

Eight patients had evidence of blush or pseudoaneurysm (PSA) on CT scan done on presentation. A splenic PSA was defined as an abnormal accumulation of contrast contained within the parenchyma of the spleen. A splenic blush was defined as any extravasation of contrast outside the parenchyma of the spleen. Splenic blush and PSA were observed in 8 out of 53 patients (15\%). PSA was observed in 3 patients $(6 \%)$ one patient with Grade III (Case 1, Table 3), two patients with Grade IV (Cases 2 and 3), while contrast blush was noted in 5 patients $(9 \%)$; two patient with grade IV and in 3 patients with grade IV injuries. Five patients out of the eight with blush or PSA had splenectomy on presentation for reasons explained above. One patient with grade III and two patients with grade IV injuries proceeded to angiography. Only one patient with Grade IV injury had evidence of contrast extravasation on angiography and was controlled successfully with proximal splenic artery embolization (Table 3).

\begin{tabular}{|l|l|l|l|l|l|l|l|}
\hline Case No. & AAST Grade & ISS & Angiography & Angiography finding & TAE & $\begin{array}{l}\text { Initial } \\
\text { plan }\end{array}$ & \multicolumn{1}{|c|}{ Treatment } \\
\hline 1 & 3 & 47 & Fes & Negative & No & NOM & No \\
\hline 2 & 4 & 18 & Yes & Negative & No & NOM & No \\
\hline 3 & 4 & 25 & Yes & Positive & Yes & TAE & No \\
\hline 4 & 4 & 13 & No & N/A & N/A & Splenectomy \\
\hline 5 & 4 & 16 & No & N/A & N/A & N/A & Splenectomy \\
\hline 6 & 5 & 38 & No & N/A & N/A & Splenectomy \\
\hline 7 & 5 & 38 & No & N/A & N/A & Splenectomy \\
\hline 8 & 5 & 29 & No & & \\
\hline
\end{tabular}

Table 3: Patients with BSI and CT scan contrast blush.

As shown in Table 4, patients who underwent splenectomy on admission (IOM) had a significantly higher ISS than patients who had NOM (ISS 24.1 vs. 16.3 respectively; p-value 0.025 ). On presentation the IOM group had also a lower Systolic Blood Pressure (SBP) of 103.9 $\mathrm{mm} \mathrm{Hg}$ vs. $122.4 \mathrm{mmHg}$ ( $\mathrm{p}=0.026$ ) observed in the NOM group.

No statistical difference was noted between the proportion of patients presenting with SBP less than 90 nor with respect to GCS or hemoglobin level (Table 3).

The length of hospital stay was higher in the splenectomy group (20 vs. 16 days) ( $\mathrm{p}=0.757)$, with a longer ICU stay of 10.3 vs. 5.9 days respectively $(\mathrm{p}=0.351)$. The need for respiratory support and the development of infectious and other complications didn't vary significantly between both groups.

\begin{tabular}{|l|l|l|l|}
\hline \multirow{2}{*}{ Variables } & IOM & NOM & \multirow{2}{*}{-value } \\
\cline { 2 - 4 } & $\mathbf{( N = 1 1 )}$ & $\mathbf{( N = 4 1 )}$ & \\
\hline ISS Mean (S.D) & $24.1(12.2)$ & $16.3(8.8)$ & 0.025 \\
\hline SBP Mean (S.D) & $103.9(19.2)$ & $122.4(23.4)$ & 0.026 \\
\hline SBP<90 (\%) & $3(30)$ & $6(14.6)$ & 0.495 \\
\hline Age & 43 & 32 & 0.14 \\
\hline GCS (S.D) & $13.4(2.9)$ & $14.1(2.3)$ & 0.485 \\
\hline
\end{tabular}


Citation: El-Rifai AY, Abi-Saad G, Qaraqe T, Farhat H, El-Hechi M, et al. (2018) Management of Blunt Splenic Injuries in the Setting of Low Trauma Volume: Challenges and Perspectives. J Trauma Treat 7: 430. doi:10.4172/2167-1222.1000430

Page 5 of 7

\begin{tabular}{|l|l|l|l|}
\hline Hemoglobin (S.D) & $12.2(2.7)$ & $13.8(2.2)$ & 0.056 \\
\hline LOS-Hospital (S.D) & $20.2(29.0)$ & $16.3(36.5)$ & 0.757 \\
\hline LOS-ICU (S.D) & $10.3(21.3)$ & $5.9(10.7)$ & 0.351 \\
\hline Respiratory Support (\%) & $4(40)$ & $10(24.4)$ & 0.962 \\
\hline Infectious Complications (\%) & $3(30)$ & $10(24.4)$ & 0.395 \\
\hline Other Complication (\%) & $0(0)$ & $6(14.6)$ & 0.468 \\
\hline Mortality (\%) & $0(0)$ & $1(2.4)$ & - \\
\hline
\end{tabular}

Table 4: Variables and outcomes per management plan, IOM vs. NOM.

Analysis of the PRBC transfusion requirements across both groups showed that NOM group had a mean transfusion for PRBC at two days of 1.1 as opposed to 5.9 units in the IOM group ( $\mathrm{p}$-value $<0.01$ ).

The transfusion requirements in the NOM group on day seven was 1.71 units of PRBCs vs. 6.8 units in the IOM group (p-value 0.001). A similar trend of blood transfusion requirements was observed across all injury grades (Table 5).

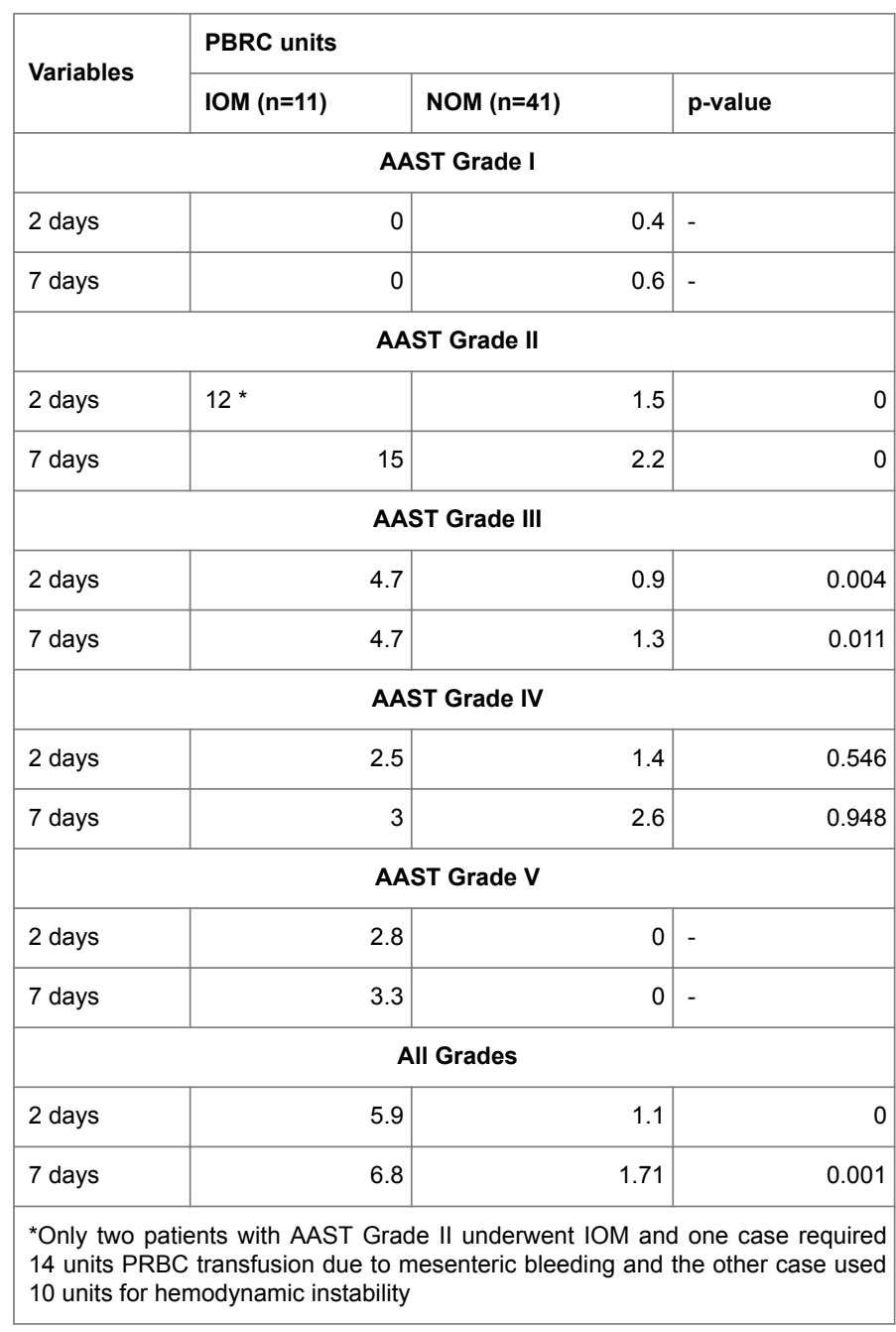

Table 5: Transfusion requirements per management plan across AAST injury grades at 2 and 7 days.

The failure rate of NOM was $12.1 \%$ (Table 6). None of the patients who had Grade I injury failed NOM while the failure rate across the different AAST injury grade II to IV was $9.1 \%, 15.4 \%$ and $22.2 \%$ respectively. One out of 53 patients died resulting in mortality rate of $1.8 \%$. The mean time duration to failure was 6 days.

\begin{tabular}{|l|l|l|l|l|}
\hline $\begin{array}{l}\text { AAST } \\
\text { Grade }\end{array}$ & IOM & NOM & Failure & $\%$ Failure \\
\hline I & 0 & 8 & 0 & 0 \\
\hline II & 2 & 11 & 1 & 9 \\
\hline III & 3 & 13 & 2 & 15.4 \\
\hline IV & 2 & 9 & 2 & 22 \\
\hline V & 4 & 0 & N/A & N/A \\
\hline Total & $11(21 \%)$ & $41(77 \%)$ & 5 & 12.1 \\
\hline
\end{tabular}

Table 6: Failure rates per AAST injury grade.

The mean age of the patients in the failed Non Operative Management (f-NOM) group was 45 years while patients who had a successful Non Operative Management (s-NOM) group had an average age of 30 years. A higher percentage of comorbidities was also observed in the f-NOM group. There was no difference in ISS, admission SBP, GCS, hemoglobin level, PRBC transfusion on day 2 between patients in the s-NOM $(n=41)$ and patients in the $\mathrm{f}-\mathrm{NOM}$ $(\mathrm{n}=5)$ groups.

Only transfusion requirement by day 7 was higher in patients in the f-NOM group. The average PRBC transfusion in this group was 3.4 as opposed to 1.7 units transfused in the s-NOM group. Outcomes of both groups didn't differ with respect to length of hospital stay, length of ICU stay, need for respiratory support and development of complications (Table 7).

\begin{tabular}{|l|l|l|l|}
\hline Variables & s-NOM (n=36) & f-NOM (n=5) & p-value \\
\hline Age & $29.9(14.3)$ & $45.0(260.5)$ & 0.05 \\
\hline Comorbidities N (\%) & $8(22)$ & $2(40)$ & 0.39 \\
\hline ISS Mean (S.D) & $16.1(8.1)$ & $17.8(13.8)$ & 0.69 \\
\hline SBP Mean (S.D) & $122.2(23.0)$ & $123.4(29.0)$ & 0.91 \\
\hline SBP<90 (\%) & $4(11)$ & $1(20)$ & \\
\hline GCS mean (S.D) & $14.2(2.1)$ & $13.6(3.1)$ & 0.59 \\
\hline Hemoglobin mean (S.D) & $13.8(2.2)$ & $13.2(1.6)$ & 0.52 \\
\hline PRBC Transfusions & & & \\
\hline 2 days mean (range) & $0.9(0-7)$ & $1.8(1-8)$ & 0.36 \\
\hline 7 days mean (range) & $0.53(0-3)$ & $2(0-4)$ & 0.005 \\
\hline LOS-Hospital mean (range) & $17.3(3-240)$ & $8.6(5-15)$ & 0.63 \\
\hline LOS-ICU mean (range) & $5.9(-60)$ & $5.2(1-13)$ & 0.88 \\
\hline Respiratory Complications N (\%) & $8(23)$ & $2(40)$ & 0.42 \\
\hline Infectious Complications N (\%) & $8(23)$ & $2(40)$ & 0.47 \\
\hline
\end{tabular}


Citation: El-Rifai AY, Abi-Saad G, Qaraqe T, Farhat H, El-Hechi M, et al. (2018) Management of Blunt Splenic Injuries in the Setting of Low Trauma Volume: Challenges and Perspectives. J Trauma Treat 7: 430. doi:10.4172/2167-1222.1000430

Page 6 of 7

\begin{tabular}{|l|l|l|l|}
\hline Other Complications N (\%) & $4(11)$ & $2(40)$ & 0.09 \\
\hline
\end{tabular}

Table 7: Differences between f-NOM and s-NOM.

Thirty-six out of 41 patients were successfully observed without requiring surgery or embolization, resulting in $87.8 \%$ successful NOM. Five patients failed NOM. Three patients had splenectomy and two patients underwent embolization.
One patient who had embolization after failing NOM required splenectomy due to continued bleeding. In total, four out of five patients $(80 \%)$ who failed NOM needed splenectomy. The total splenectomy rate in the cohort of patients admitted with BSI is 14 out of $53(26 \%)$. The characteristics and outcomes of the five patients who failed non operative management and the timing of their failure are shown in Table 8.

\begin{tabular}{|c|c|c|c|c|c|c|c|c|c|c|}
\hline & Age & $\begin{array}{l}\text { Hemodynamic } \\
\text { stability }\end{array}$ & ISS & $\begin{array}{l}\text { AAST } \\
\text { grade }\end{array}$ & Major issue & Reason for failure & $\begin{array}{l}\text { Time to } \\
\text { failure } \\
\text { in days }\end{array}$ & Treatment & Complication & $\begin{array}{l}\text { Year of } \\
\text { injury }\end{array}$ \\
\hline P1 & 36 & stable & 14 & III & $\begin{array}{lr}\text { Liver laceration, } \\
\text { missed r contrast } \\
\text { extravasation from } \\
\text { the } & \text { splenic } \\
\text { laceration. }\end{array}$ & $\begin{array}{l}\text { Hemodynamic } \\
\text { decompensation } \\
\text { from bleeding }\end{array}$ & 6 & splenectomy & ARDS & 2011 \\
\hline P2 & 56 & Stable & 13 & III & $\begin{array}{l}\text { Large } \\
\text { hemoperitoneum, } \\
\text { perihepatic and } \\
\text { perispslenic Multiple } \\
\text { rib fracture }\end{array}$ & $\begin{array}{l}\text { Hemodynamic } \\
\text { decompensation } \\
\text { from bleeding }\end{array}$ & 3 & splenectomy & none & 2011 \\
\hline P3 & 28 & Stable & 4 & II & no & $\begin{array}{l}\text { Drop in } \\
\text { hemoglobin }\end{array}$ & 10 & splenectomy & none & 2011 \\
\hline P4 & 20 & Unstable & 41 & IV & $\begin{array}{l}\text { Hypotnesiver on } \\
\text { arrival. Multiple } \\
\text { injuries including } \\
\text { grade III liver } \\
\text { laceration, major } \\
\begin{array}{l}\text { lung contusion and } \\
\text { Lefort III fracture }\end{array}\end{array}$ & $\begin{array}{l}\text { Bleeding drop in } \\
\text { hemoglobin }\end{array}$ & 4 & Splenectomy & $\begin{array}{l}\text { RefractoryHypoxemia } \\
\text { secondary to ARDS. } \\
\text { Death }\end{array}$ & 2013 \\
\hline P5 & 85 & stable & 17 & IV & $\begin{array}{l}\text { Small } \\
\text { Pseudoaneurysm }\end{array}$ & Follow up CT & 3 & TAE & None & 2015 \\
\hline
\end{tabular}

Table 8: Characteristics and outcomes of the five patients who failed NOM.

\section{Conclusion}

In a low trauma volume University Hospital, a trial of non operative management for high grade blunt splenic injures (AAST Grade III-IV) with no evidence of ongoing bleeding is safe in the presence of an established trauma team and close monitoring. Further studies are needed to clarify whether this category of patients will benefit from embolization when the CT shows a pseudoaneurysm but angiography is normal. Finally in a setting like ours we recommend the implementation of written protocols that take in account the multidisciplinary approach required for the accurate and timely management of splenic injury.

\section{References}

1. Rowell SE, Biffl WL, Brasel K, Moore EE, Albrecht RA, et al. (2017) Western trauma association critical decisions in trauma: Management of adult blunt splenic trauma-2016 updates. J Trauma Acute Care Surg 82: 787-793.

2. Rosati C, Ata A, Siskin GP, Megna D, Bonville DJ, et al. (2015) Management of splenic trauma: A single institution's 8-year experience. Am J Surg 209: 308
3. Harbrecht BG, Zenati MS, Ochoa JB, Puyana JC, Alarcon LH, et al. (2007) Evaluation of a 15-year experience with splenic injuries in a state trauma system. Surgery 141: 229-238.

4. Mebius Re, Kraal G (2005) Structure and function of the spleen. Nat Rev Immunol 5: 606-616.

5. Peitzman AB, Heil B, Rivera L, Federle MB, Harbrecht BG, et al. (2000) Blunt splenic injury in adults: Multi-institutional study of the eastern association for the surgery of trauma. J Trauma Infect Crit Care 49: 177189.

6. Velmahos P (2010) Management of the most severely injured spleen:A multicenter study of the research consortium of new england centers for trauma. Arch Surg 145: 456-460.

7. Requarth JA, D'Agostino RB, Miller PR (2011) Nonoperative management of adult blunt splenic injury with and without splenic artery embolotherapy: A meta-analysis. J Trauma 71: 898-903.

8. Sabe AA, Claridge JA, Rosenblum DI, Lie K, Malangoni MA, et al. (2009) The effects of splenic artery embolization on nonoperative man- agement of blunt splenic injury: A 16-year experience. J Trauma Acute Care Surg 67: 565-572.

9. Miller PR, Chang MC, Hoth JJ, Mowery NT, Hildreth AN, et al. (2014) Prospective trial of angiography and embolization for all grade iii to $\mathrm{v}$ blunt splenic injuries: Non-operative management success rate is significantly improved. J Am Coll Surg 218: 644-648. 
Citation: El-Rifai AY, Abi-Saad G, Qaraqe T, Farhat H, El-Hechi M, et al. (2018) Management of Blunt Splenic Injuries in the Setting of Low Trauma Volume: Challenges and Perspectives. J Trauma Treat 7: 430. doi:10.4172/2167-1222.1000430

Page 7 of 7

10. Skattum J, Naess PA, Eken T, Gaarder C (2013) Refining the role of splenic angiographic embolization in high-grade splenic injuries. J Trauma Acute Care Surg 74: 100-104.

11. Bhullar IS, Frykberg ER, Siragusa D, Chesire D, Paul J, et al. (2012) Selective angiographic embolization of blunt splenic traumatic injuries in adults decreased failure rate of nonoperative management. J Trauma 72: 1127-1134.

12. Bhullar IS, Frykberg ER, Tepas JJ, Siragusa D, Loper T, et al. (2013) At first blush: Absence of computed tomography contrast extravasation in grade IV or $\mathrm{V}$ adult blunt splenic trauma should not preclude angioembolization.

13. Gaarder C, Dormagen JB, Eken T, Skaga NO, Klow NE, et al. (2006) Nonoperative management of splenic injuries: Improved results with angioembolization. J Trauma 6: 192-198.

14. Chastang L, Bège T, Prudhomme M, Simonnet AC, Herrero A, et al. (2015) Is non-operative management of severe blunt splenic injury safer than embolization or surgery? Results from a French prospective multicenter study. J Visc Surg 152: 85-91.

15. Al-Qahtani MS (2004) The pattern and management outcomes of splenic injuries in the Assir region of Saudi Arabia. West Africa Journal of Medicine p: 23.

16. Saurabh G, Kumar S, Gupta A, Mishra B, Sagar, et al. (2011) Splenic trauma-our experience at a level I Trauma Center. Ulus Travma Aci Cerrahi Derg 17: 238-242.

17. Erdogan D (2001) Traumatic Splenic Injuries, Turkish Journal of Trauma \& Emergency Surgery 7: 1

18. Moore EE, Cogbill TH, Jurkovich GJ (1995) Organ injury scaling: Spleen and liver. J Trauma 38: 223.

19. Smith J, Armen S, Cook CH, Martin LC (2008) Blunt splenic injuries. Have we watched long enough? J Trauma 64:656-663; Discussion 64: 663-665.

20. Smith SR, Morris L, Spreadborough S (2017) Management of blunt splenic injury in a UK major trauma centre and predicting the failure of non-operative management: A retrospective, cross-sectional study. Eur J Trauma Emerg Surg 2: 1.

21. Zarzaur BL, Kozar RA, Fabian TC, Coimbra R (2011) A survey of American Association for the Surgery of Trauma member practices in the management of blunt splenic injury. J Trauma 70: 1026-1031.
22. Harbrecht P (2007) Evaluation of a 15-year experience with splenic injuries in a state trauma system. Surgery. 141: 229-238.

23. Harbrecht BG, Ko SH, Watson GA, Forsythe RM, Rosengart MR, et al. (2007) Angiography for blunt splenic trauma does not improve the success rate of nonoperative management. J Trauma 63: 44-49.

24. Zarzaue BL, Kozar R, Myers JG, Claridge JA, Scalea TM, et al. (2015) The splenic injury outcomes trial: An American Association for the Surgery of Trauma multi-institutional study. J trauma Acute Care Surg 79: 335-342.

25. Ekeh AP, Khalaf S, Ilvas S, Kauffman S, Walusimbi M, et al. (2013) Complications arising from splenic artery embolization: a review of an 11-year experience. Am J Surg 205: 250-254.

26. Weinberg JA, Lockhart ME, Parmar AD (2010) Computed tomography identification of latent pseudoaneurysm after blunt splenic injury: pathology or technology? J Trauma 68: 1112-1116.

27. Muroya T, Ogura H, Shimizu K (2013) Delayed formation of splenic pseudoaneurysm following nonoperative management in blunt splenic injury: A multi-institutional study in Osaka, Japan. J Trauma Acute Care Surg Volume 75: 417-450.

28. Smith J, Armen S, Cook CH, Martin LC (2008) Blunt splenic injuries: Have we watched long enough? J Trauma 64: 656-665.

29. Moore FA, Davis JW, Moore EE, Cocanour CS, West MA, et al. (2008) Western trauma Association critical decisions in trauma: Management of adult blunt splenic trauma. J Trauma 65: 1007-1011.

30. Olthof DC, Joosse P, Van der Vlies CH, De Haan RJ, Goslings JC, et al. (2013) Prognostic factors for failure of nonoperative management in adults with blunt splenic injury: A systematic review. J Trauma Acute Care Surg 74: 546-557.

31. Cirocchi R, Boselli C, Corsi A, Farinella E, Listorti C, et al. (2013) Is nonoperative management safe and effective for all splenic blunt trauma? A systematic review. Crit Care 17: 185.

32. Velmahos GC, Toutouzas KG, Radin R, Chan L, Demetriades D, et al. (2003) Nonoperative treatment of blunt injury to solid abdominal organs - A prospective study. Arch Surg 138: 844-849.

33. Richardson JD (2005) Changes in the management of injuries to the liver and spleen. J Am Coll Surg 200: 670-676.

34. Peitzman AB, Harbrecht BG, Rivera L, Heil B (2005) Failure of observation of blunt splenic injury in adults: variability in practice and adverse consequences. J Am Coll Surg 201: 179-187. 